\title{
DALL'AUTOBIOGRAFIA AL DOCUMENTO. \\ IL GRAPHIC NOVEL TRA MEMORIA ARCHIVIATA E \\ SVELAMENTO DELL'ILLUSIONE IN ANNE FRANK. THE \\ ANNE FRANK HOUSE AUTHORIZED \\ GRAPHIC BIOGRAPHY
}

\section{FROM THE AUTOBIOGRAPHY TO THE DOCUMENT. THE GRAPHIC NOVEL BETWEEN ARCHIVED MEMORY AND UNVEILING OF ILLUSION IN ANNE FRANK. THE ANNE FRANK HOUSE AUTHORIZED GRAPHIC BIOGRAPHY}

\author{
Cristina GRECO \\ Università della Sapienza di Roma \\ cristina.greco@uniroma1.it
}

\begin{abstract}
Questo contributo intende indagare le strategie di costruzione dell'effetto di realtà e di verità nel graphic novel. Attraverso l'analisi di Anne Frank. La biografia a fumetti, si proporrà una riflessione sugli elementi costitutivi dell'atto di testimoniare, sui concetti di documento e memoria archiviata elaborati da Paul Ricoeur (2000), e sullo sforzo di un certo romanzo a fumetti di far sì che ciò di cui si racconta venga riconosciuto come autentico. Si tratta di riflettere sul rapporto tra la costruzione di un'illusione fittizia e lo svelamento dell'artificiosità di un testo complesso, questione che mette in gioco il discorso sulcredere e sul contratto fiduciario.
\end{abstract}

Parole chiave: Auto/biografia. Documento. Graphic novel. Autenticità. Memoria collettiva. Anne Frank. La biografia a fumetti. Sid Jacobs ed Ernie Cólon.

Abstract: This paper aims to investigate the discursive strategies that produce the effect of sense of reality and truth in the graphic novel. Through the analysis of a representative graphic novel, The Anne Frank House Authorized Graphic Biography, this paper proposes a reflection on the constitutive elements of the act of witnessing, in view of Ricoeur's concepts of document and archived memory (2000) and on the effort to ensure that what it is said will be recognized as authentic. From this perspective, we can reflect on the relationship between the construction of a fictional illusion and the unveiling of the artificiality of a complex text, characterized by a heterogeneity of elements, which calls into question the discourse on truth-believing and fiduciary contract.

Key Words: Auto/biography. Document. Graphic novel. Authenticity. Collective memory. The Anne Frank House Authorized Graphic Biography. Sid Jacobs and Ernie Cólon. 
Preso nel fascio delle questioni,

il documento non cessa di allontanarsi dalla testimonianza. Niente è, in quanto tale, documento, anche se qualsiasi residuo del passato è potenzialmente traccia. Paul Ricoeur (2000, trad. it. 2003: 252)

\section{PREMESSA: DOCUMENTO E TESTIMONIANZA}

Quando Paul Ricoeur elabora il concetto di "testo orfano" inserendolo nel quadro di una riflessione sulla funzione testimoniale del documento d'archivio, è per individuarne la forza comunicativa e gli effetti di una sua traduzione in una diversa testualità (2000). In La memoria, la storia, l'oblio, Ricœur, con un ragionevole distacco in termini non solo temporali ma anche teorici da Tempo e racconto II (1984), espone una riflessione sulla rappresentazione storica, e dedica un capitolo alla memoria archiviata, ponendo sin dall'inizio, come suo presupposto, un punto di origine, ovvero la testimonianza.Egli compie un percorso che dal momento in cui qualcuno dice qualcosa, dunque dall'iscrizione dell'atto testimoniale, procede fino alla questione della nascita dell'archivio, raccolto, conservato e consultato e, del momento in cui, la testimonianza, una volta uscita dagli archivi, non solo si trova al cospetto di altre testimonianze anche divergenti tra loro, ma viene assorbita in una massa di documenti non costituita unicamente da testimonianze.

Parlare oggi di riuso del documento d'archivio vuol dire considerare quella condizione di trasversalità caratterizzante il nuovo rapporto tra i media. Non si tratterebbe infatti di porre una distinzione tra il vecchio e il nuovo, così come non è sufficiente parlare di incontro e sovrapposizione in termini di uso delle tecnologie, ma di un sistema traduttivo e costantemente trasformativo, entro cui sono i contenuti a modificarsi al fine di sfruttare al meglio tutte le possibilità offerte da un sistema dinamico fatto di intrusioni semiotiche,mediate da confinientro cui “l'eterogeneità strutturale dello spazio semiotico forma riserve di processi dinamici ed è uno dei meccanismi di elaborazione di nuova informazione all'interno della sfera" (Lotman 1984, trad. it. 1992: 16).

Scegliendo il graphic novel come oggetto di ricerca, e in particolare quello che si fa portatore di un incontro tra i generi, dalla biografia al racconto autobiografico e di testimonianza, mi sono posta l'obiettivo di esaminare, attraverso gli strumenti analitici della semiotica greimasiana, gli effetti di senso generati in seno a una testualità emergente, che sempre più ingloba e integra la prova documentale, facendosi essa stessa documento, ed esasperando i suoi tratti costitutivi. Per fare questo, procederò alla luce di alcuni dei concetti chiave presenti nel pensiero ricoeuriano, con particolare riferimento 
alle nozioni di documento, ai problemi dell'autodesignazione del soggetto testimone e della crisi della testimonianza, tenendo a mente l'idea di veridizione.

Seguardiamoalle più recenti produzioni culturali, spostandociverso quellediconsumo e popolari, vediamo come le immagini di archivio, siano esse documenti di famiglia, vecchie carte istituzionali, manoscritti, testimonianze ecc. ricorrano nella letteratura, nel cinema, e nel graphic novel in modo più frequente rispetto alle esperienze del passato. Nel caso in cui non vi sia un innesto dell'immagine fotografica di un documento, esso è rielaboratocome figura o tema a livello verbale o visivo, o verbo-visivo. Pensiamo alla raffigurazione dellepagine ingiallite del diario di un soldato, come in On les aura!', oppure alla ricerca cromatica di un effetto seppia nel disegno delle carte d'identità dei profughi di Fiume, come in Palacinche. Storia di un'esule fiumana ${ }^{2}$. Sono solo alcuni dei casi in cui gli elementi costitutivi del documento rientrano come citazione in quel fumetto che si fa "maestro"di traduzione, per via del suo carattere composito e sincretico, e diventa esso stesso testimone attraverso la costruzione di un'istanza soggettiva.

Porre in questi termini la questione del racconto del sé e dell'altro, quindi del racconto autobiografico e biografico, e dell'uso della fonte documentale nel graphic novel, vuol dire spostare il centro della discussione da uno studio delle dinamiche di funzionamento del testo a uno studio della sua capacità di dialogo e di spostamento sui confini della sfera culturale, dunque, nel quadro di una semiotica della cultura.

\section{L'AUTENTICO E LA RI-COSTITUZIONE DELL'IO. DA ANNE FRANK. THE ANNE FRANK HOUSE AUTHORIZED GRAPHIC BIOGRAPHY ${ }^{3}$}

Oggi il graphic novel occupa un posto centrale nell'elaborazione di tematiche di interesse collettivo. Dal romanzo a fumetti autobiografico al graphic journalism, dal diario di viaggio alle biografie a fumetti, sempre più si assiste a uno sviluppo crescente e diversificato delle modalità di messa in atto della testimonianza. II ruolo svolto dal reportage a fumetti, come Palestine di Joe Sacco ${ }^{4}$, in qualità di testimonedi una cronaca che si fa storia, si affianca a una ricerca e a un inglobamento delle fonti primarie della memoria collettiva. Dall'altra parte però la specificità investigativa del discorso biografico e la costruzione della soggettività e del dire vero del discorso dell'lo testimone nel racconto autobiograficopongono le basi per un ripensamento

1 On les aura! realizzato dall'illustratore di libri per l'infanzia, Barroux, e narra, attraverso la messinscena di un diario, le vicende di un soldato sconosciuto durante la Prima Guerra Mondiale. Palacinche è un graphic novel realizzato da Caterina Sansone e Alessandro Tota.

3 Per l'analisi, è stata impiegata la versione italiana del graphic novel.

4 Per un approfondimento e un'analisi di Palestina, cf. Greco (2009). 
del concetto stesso di documento. Ciò ha investito anche il web, con reportage a fumetti e graphic novel autobiografici nati appositamente per esser fruiti online, che danno vita, in alcuni casi, a un nuovo tipo di narrazione, caratterizzata dall'esperienza immersiva e fusionale vissuta dal lettore/spettatore.La costruzione di un effetto di verità nel graphic novel può essere allora esaminata proprio sulla base del conflitto generato da queste diverse posizioni, permettendoci di lavorare sul rilevamento delle modalità enunciative della veridizione (Greimas, 1983).

Per la capacità di ridefinizione dei suoi tratti costitutivi e di dialogo con altre forme espressive, ad esempio la fotografia e il cinema, il graphic novel si attesta come spazio di valorizzazione delle convergenze sia tra le diverse sostanze dell'espressione, sia tra i diversi media. In questa prospettiva, se in passato ho lavorato, ad esempio, sulla problematica del credere tra fumetto e reportage fotografico nell'analisi de Le Photographe di Guibert, Lefevre e Lemercier, e sulla costruzione dell'effetto di reale nel diario di viaggio, nell'analisi di On les aura! di Barroux (cf. Greco, 2014), qui analizzerò un'opera in particolare, che ritengo esemplare rispetto alle premesseavanzate. Si tratta di Anne Frank. The Anne Frank House Authorized Graphic Biography di Sid Jacobs ed Ernie Cólon.

L'eccezionalità dell'opera è data da più fattori. Innanzitutto essa si pone a metà strada tra la ricostruzione biografica e l'adattamento letterario. La prima parte ricostruisce la storia della famiglia Frank, a partire dal suo unico sopravvissuto, Otto Frank; la seconda, evidenzia le tappe fondamentali del Diario di Anne, adattandolo a una forma nuova, quella del fumetto. In secondo luogo, la rappresentatività dell'opera è data dalla sua aderenza alla problematica sollevata dall'innesto del documento e dal suo funzionamento all'interno del racconto di finzione. Essa implica un uso della fonte documentale, che va al di là della sua consultazione, per innestarla in un altro testo, costituito da un insieme di connessioni tra il racconto dei fatti che si intendono realmente accaduti e la ricostruzione più o meno fittizia degli eventi.

Infine, la sua capacità di produrre senso è data dallegame che intrattiene con gli altri testi della semiosfera. Non a caso già il sottotitolo funge da prima indicazione alla portata intertestuale del graphic novel, riportandoci alla questione dell'autenticoe del credere-vero del destinatario, sollecitati dal marcatore "authorized", che implica non solo un poter-fare concesso da un soggetto dotato di per sé di credibilità (dall'Anne Frank House), ma anche un atto di riconoscimento dell'essere (Fig. 1). 


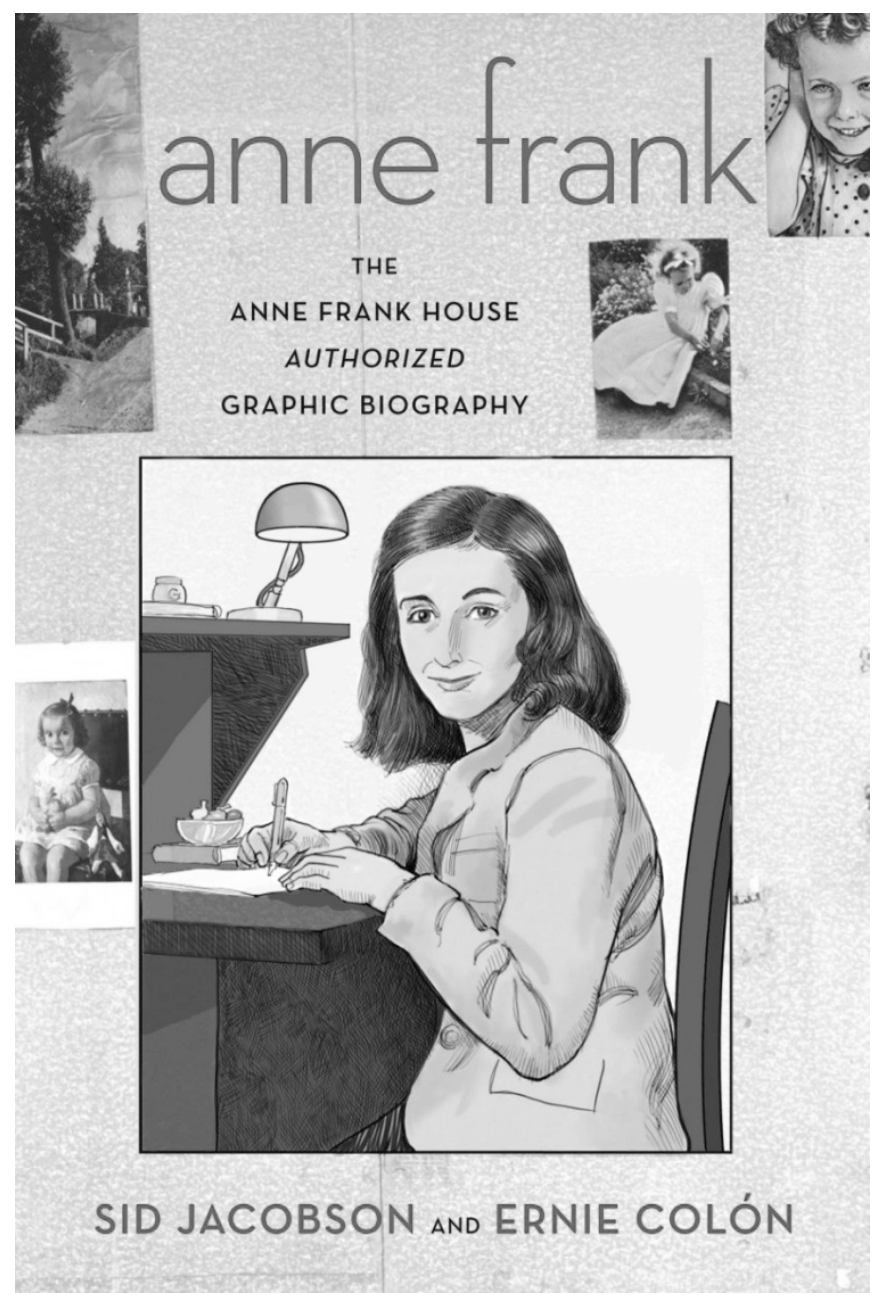

Fig. 1 Anne Frank. The Anne Frank House Authorized Graphic Biography.

Cover $\odot$ Hill and Wang (2010)

L'opera è divisa in dieci capitoli (Un promettente inizio; Annelies Marie Frank; L'ascesa del nazismo; Amsterdam; Sotto il dominio tedesco; Il diario; Gli otto del rifugio; Anno nuovo; Scoperti; La Storia va avanti). In apertura ritroviamo la prefazione, che nell'edizione italiana è a opera di Sergio Luzzatto, docente di Storia Moderna all'Università di Torino, e in chiusura al volume una Cronologia, i Consigli di lettura e i Ringraziamenti. La compresenza di diversi elementi strutturali e stilistici lo rende un testo articolato, eterogeneo e plurale. Ogni capitolosi apre con una splash page ${ }^{5}$ contenente il titolo e una tavola che, dal quattro in poi, è caratterizzata dal chiaro/

5 Con l'espressione "spash page" si intende indicare la pagina del fumetto interamente occupata da un'immagine priva di riquadri. 
scuro, che richiama il tema della memoria, in opposizione alla policromia delle tavole, che indica un tempo attuale. Essa funge da incipit, quasi a indicare al lettore quanto dovrà aspettarsi dal procedere della lettura, ma al tempo stesso crea un continuum rispetto a quanto incontrato nel capitolo precedente. Tali espedienti verbo-visivi hanno la funzione di rendere omogeneo e lineare il testo, di fronte alla complessità che lo distingue, data non solo dalla diversità delle componenti (ad esempio, alcune tavole richiedono di esser lette in orizzontale), ma anche dall'innesto di elementi che potrebbero creare una rottura della narrazione. Anche qui essi svolgono la funzione che altrove, nell'analisi di Le Photographe, ho indicato come "pre-memoria", ponti tra ciò che il lettore ha visto e ciò che vedrà (cf. Greco, 2014: 122). In Anne Frank, nella tipologia genettiana (1987), I'istanza prefativa ha una funzione metadiscorsiva:dichiara quelli che sono gli obiettivi del testo, ne esplicita le responsabilità e le intenzioni dell'autore, suggerisce gli indizi per una prima possibile interpretazione. In oltre, in una riflessione sulla dimensione sociale della discorsività legata al ricordo di Anne, come urgenza presso le nuove generazioni, essa fa emergere i valori assiologici che sottostanno al testo, quelli della verità, dell'autenticità, della memoria: ["Se a tutto ciò si aggiungono le innovazioni tecnologiche dell'ultimo ventennio e il loro impatto sui consumi culturali sulle nuove generazioni, si può intuire perché la Casa di Anne Frank abbia scelto di promuovere questa graphic biography: così da rendere la storia di Anne "accessibile a un pubblico il più largo possibile"'"]. Fanno seguito le dediche dei due autori, reduci da un graphic noval sull'11 settembre $2001^{6}$. II disegno realistico, cifra stilistica di Ernie Cólon, è accompagnato da dialoghi e didascalie che, in alcuni passaggi, sembra ricalchino fedelmente le fonti storiche.Già nella tavola di apertura al primo capitolo"Un promettente inizio" (Fig. 2), ritroviamo un débrayage annidato, nella presenza di indici spazio-temporali: "Edith e Otto Frank, il giorno del loro matrimonio, 12 maggio 1925, ad Aquisgrana, in Germania". Continua nella pagina successiva, nella prima vignetta, dove il narratore extradiegetico a livello verbale recita: ["Nel maggio del 1944 Anne Frank scriveva dei suoi genitori nel suo amato diario..."].

6 11/09 è il reportage a fumetti realizzato dai due autori, che racconta il prima, il durante e il dopo l'attentato alle Twin Towers. 


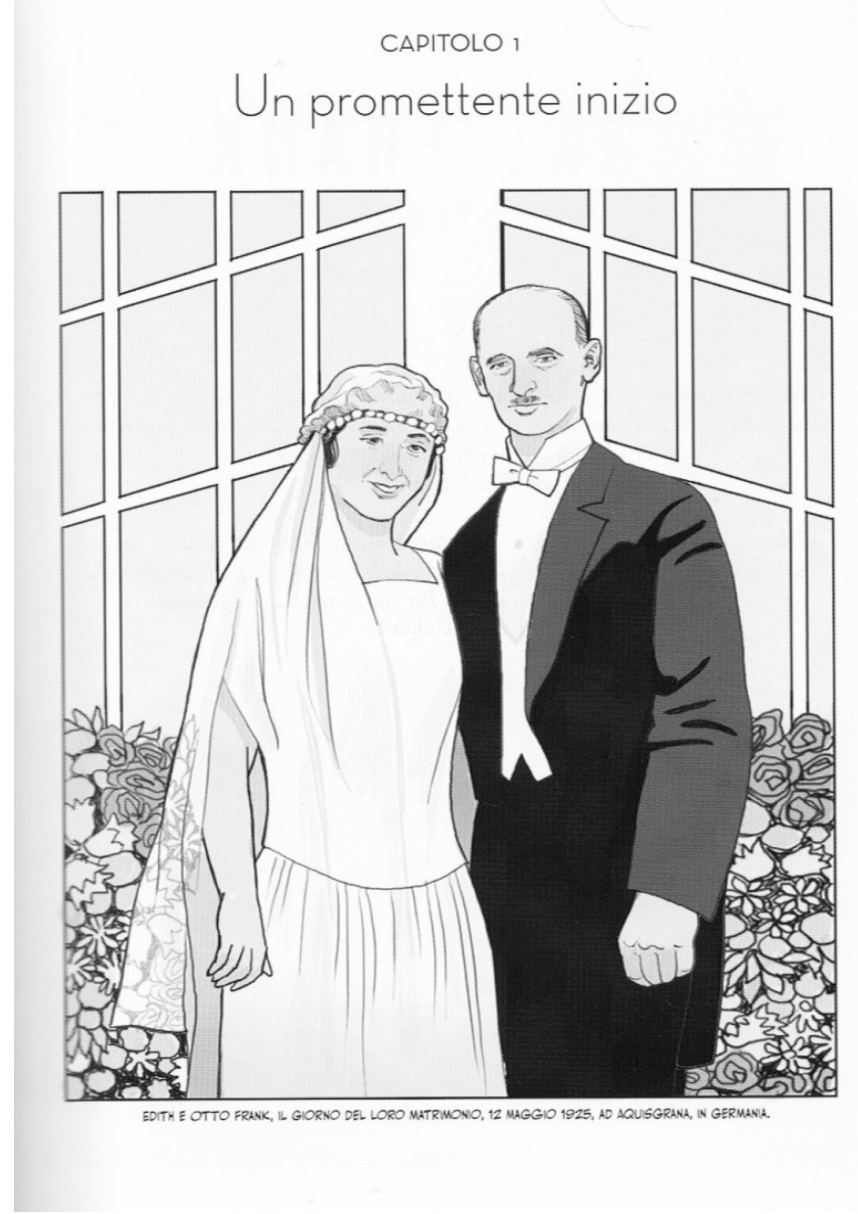

Fig. 2. Anne Frank. The Anne Frank House Authorized Graphic Biography. Tavola p. 11 (c) Rizzoli Lizard (2010).

Analizzare un fumetto richiede di porre attenzione sulla sua specificità, data dal suo essere un testo sincretico per eccellenza, per cui non è possibile ignorare le varie sostanze dell'espressione che lo compongono, ma in particolare non esaltare i significati che produce la relazione verbo-visiva. Difatti, restando sulla prima vignetta, se il testo verbale è attivatore di un débrayage attoriale, spaziale e temporale, il testo visivo opera inversamente, generando, forse con una delle più comuni strategie testuali impiegate dal fumetto, in particolare da quello documentaristico e autobiografico, un embrayage enunciazionale e un effetto di senso ${ }^{7}$ di implicazione del lettore (Fig. 3).

7 Per un approfondimento sul concetto di effetto di senso in prospettiva semiotica, vedi il capitolo ottavo, "L'efficacia del testo. Effetti e affetti nella semiosi" in Pezzini (2007). 


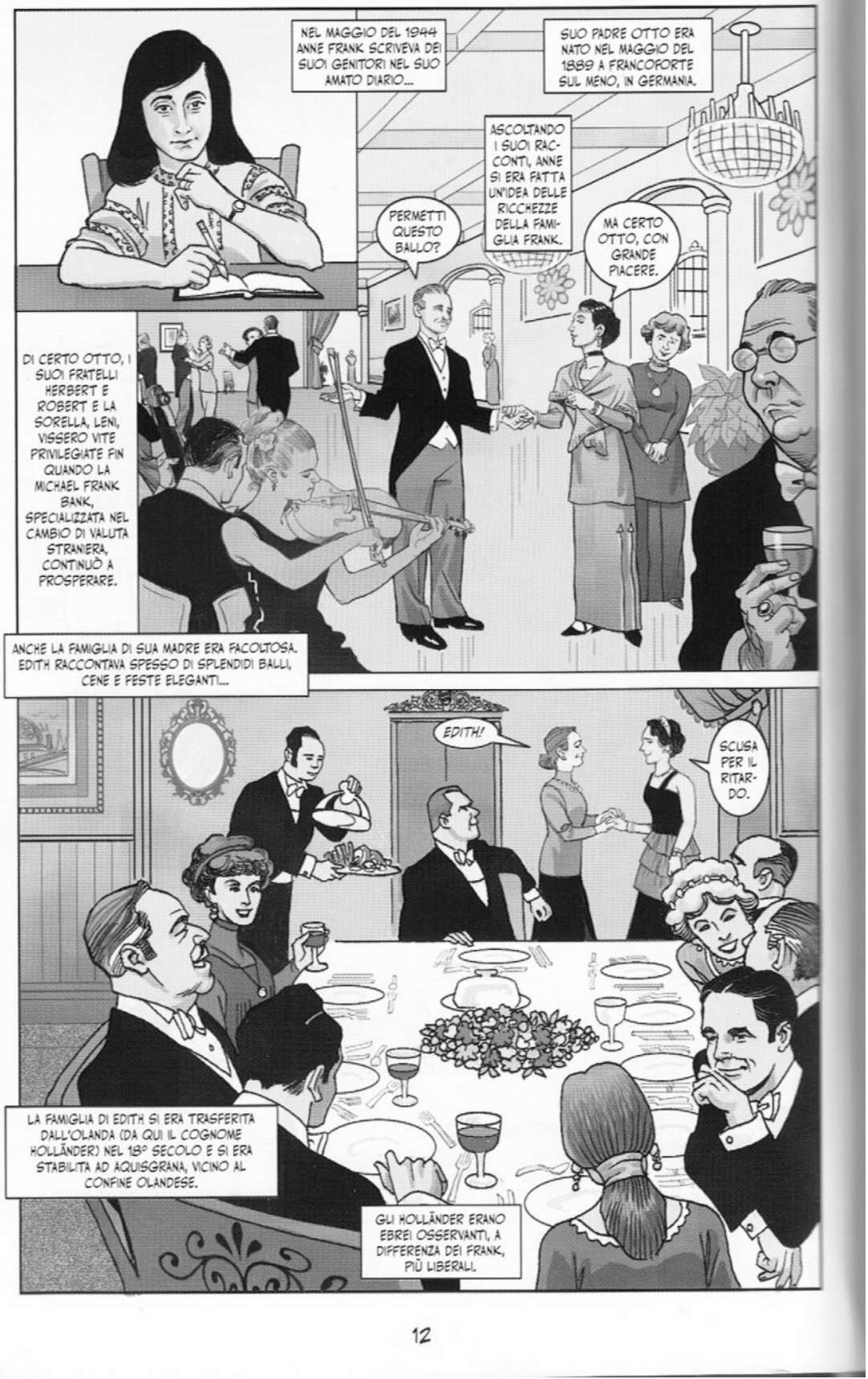

Fig. 3. Anne Frank. The Anne Frank House Authorized Graphic Biography. Tavola p. 12 ○ Rizzoli Lizard (2010). 
Se il primo, il testo verbale, narra il momento della scrittura come un tempo lontano dal presente del lettore, il secondo, quello visivo, racconta un tempo presente, e uno spazio, quello dell'enunciazione. È importante soffermarsi sulla prima vignetta, poiché essa ci spinge oltre i limiti di quello che abbiamo definito un testo che opera un riuso del documento, in quanto si serve del dispositivo dellimmaginazione e lo fa disponendo a livello visivo le marche dell'enunciazione. Lo sguardo di Anne è rivolto al posto occupato da colui che osserva la tavola, generando un effetto di chiamata in causa dell'enunciatario e di sua iscrizione all'interno dell'enunciato, nella relazione io/ tu. Si tratta di una modalità metadiscorsiva, in cui il fumetto esplicita quelle che sono le sue potenzialità, e fissa il soggetto veritiero del discorso sui fatti narrati: non il diario, ma l'lo da cui è costituito e che qui si ri-costituisce nella ricerca di un nuovo patto.Tutti questi elementi generano un effetto di reale (Barthes, 1968) e innescano un patto basato sul racconto pseudoautobiografico (Ricoeur 1984, trad. It. 1999: 25), alla cui base vi è il credere-vero.

Le vignette successive, che, a differenza di quelle sulle quali mi soffermerò perché rappresentative rispetto alle ipotesi avanzate, non possiamo esplorare, restituiscono quella relazione coerente tra il visivo e il verbale, di cui beneficerà la linearità della narrazione, salvo poi essere interrotta nella tavola successiva, che suggerisce una diversa pratica di lettura, e interrompe il racconto.In questa sono riprodotti gli alberi genealogici delle famiglia Frank e Holländer (Fig. 4).

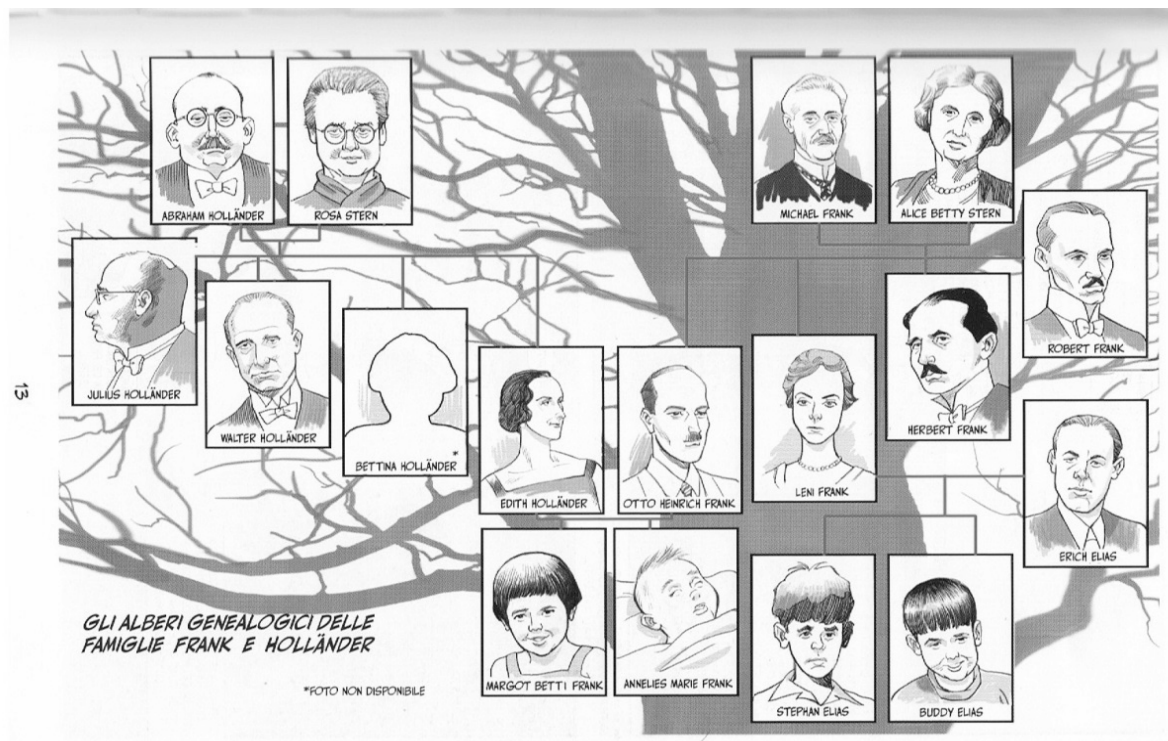

Fig. 4. Anne Frank. The Anne Frank House Authorized Graphic Biography. Tavola p. 13 (c) Rizzoli Lizard (2010). 
Si tratta di un disegno che simula la fotografia, figurativizza i temi della testimonianza e della memoria, nonché del documentale,ma vi aggiunge un elemento nuovo, l'asterisco in basso nella tavola, che investe la modalità metadiscorsiva e dunque esplicativa: ["*Foto non disponibile"]. Seguiamo le vicende della famiglia di Anne, precedenti all'evento drammatico, in un'alternanza tra le scene che ritraggono la quotidianità e scene incentrate sulla dimensione politica e di cronaca delle vicende legate all'ascesa del nazismo.

Inoltre il racconto della vita dei Frank sotto il dominio tedesco è inframezzata da vignette che presentano caratteri assimilabili sul piano verbale al saggio storico, presentato in alcuni casiattraverso il lessema"Istantanea", che genera un effetto di sospensione, e su quello visivo al documentario socio-politico (trad. it. 2010: 46). Inoltre a livello plastico, alcune di queste vignette presentano dei fattori di differenza rispetto alla totalità del testo e all'insieme delle tavole, che si manifesta in linee irregolari, ombra più nette, che sottolineano la dimensione disforica di un ricordo angoscioso,un aspetto cromatico differente dello sfondo dei riquadri, che diventa giallo o color seppia,e di alcune vignette in bianco e nero. Vi sono poi, nel quinto capitolointitolato "Sotto il dominio tedesco", tavole in cui le Istantanee riportano cartine geografiche, corredate da una legenda - che indica gli alleati e i Paesi dell'Asse dopo l'attacco di Pearl Harbour -, e da un titolo, che ne riporta l'anno di riferimento: ["Istantanea: gli stati combattenti (1942)"]. Anche qui la composizione della tavola richiede una lettura orizzontale (trad. it. 2010: 68). Anche la tavola successiva (Fig. 5) risulta significativa, poiché l'Istantanea presenta per la prima volta un documento d'archivio che, come il testo verbale enuncia, è "la lista con il numero degli ebrei presenti nei vari paesi europei che l'esercito tedesco si preparava ad affrontare". L'esigenza di soffermarsi su questa tavola è data sia dal fatto che essa racchiude i diversi fattori che abbiamo incontrato sinora - dall'interruzione della narrazione alla diversa resa plastica e figurativa, nonché all'alternanza degli stili di scrittura - sia dalla peculiare organizzazione degli elementi interni alla tavola, dunque della sua componente topologica. Difatti, se ci soffermiamo sulle caratteristiche verbo-visive di ogni tavola, possiamo riconoscere una disposizione "a incrocio". La quarta vignetta è il seguito della prima, mentre la seconda dialoga con la terza. Ciò si evince sia a livello cromatico, sia perché riconosciamo un'isotopia tematico-figurativa parziale. Tuttavia essi attivano differenti effetti di senso. Tra la prima e la quarta ritroviamo un effetto di soggettivazione, dato sul piano visivo dallo sguardo rivolto al posto che dovrebbe essere occupato dal lettore (v. 1), e dalla disposizione dei personaggi, nonché dalla fuoriuscita di una delle figura dalla cornice del riquadro, che sembra suggerire la presenza di un quarto interlocutore: il simulacro del lettore (v. 4). Tra la seconda e la terza vignetta, invece, accomunate sul piano cromatico dalla predominanza dei toni del grigio,la lettura del documento, che simula il documento dattiloscritto, e così facendodetermina lo statuto veridittivo del discorso enunciato, suggerisce la presenza di un osservatore ( $\mathrm{v}$. 
2), e si oppone all'effetto di soggettivazione generato dallo sguardo che il personaggio rivolge al posto che dovrebbe essere occupato dal lettore, chiamandolo in causa. Inoltre, la vittima implicita nella seconda vignetta è manifestae rivelata nella terza vignetta a livello figurativo dall'espressione del volto del personaggio in primo piano (dominato), che si oppone al soldato delle SS alle sue spalle (dominante), e dal filo spinato, che indica un non poter fare; il testo verbale conferma l'isotopia tematico-figurativo dominante/ dominato - del "noi" che si oppone all'"estraneo", "condannavamo tutti gli ebrei" -, e il valore disforico nel lessema "morte".

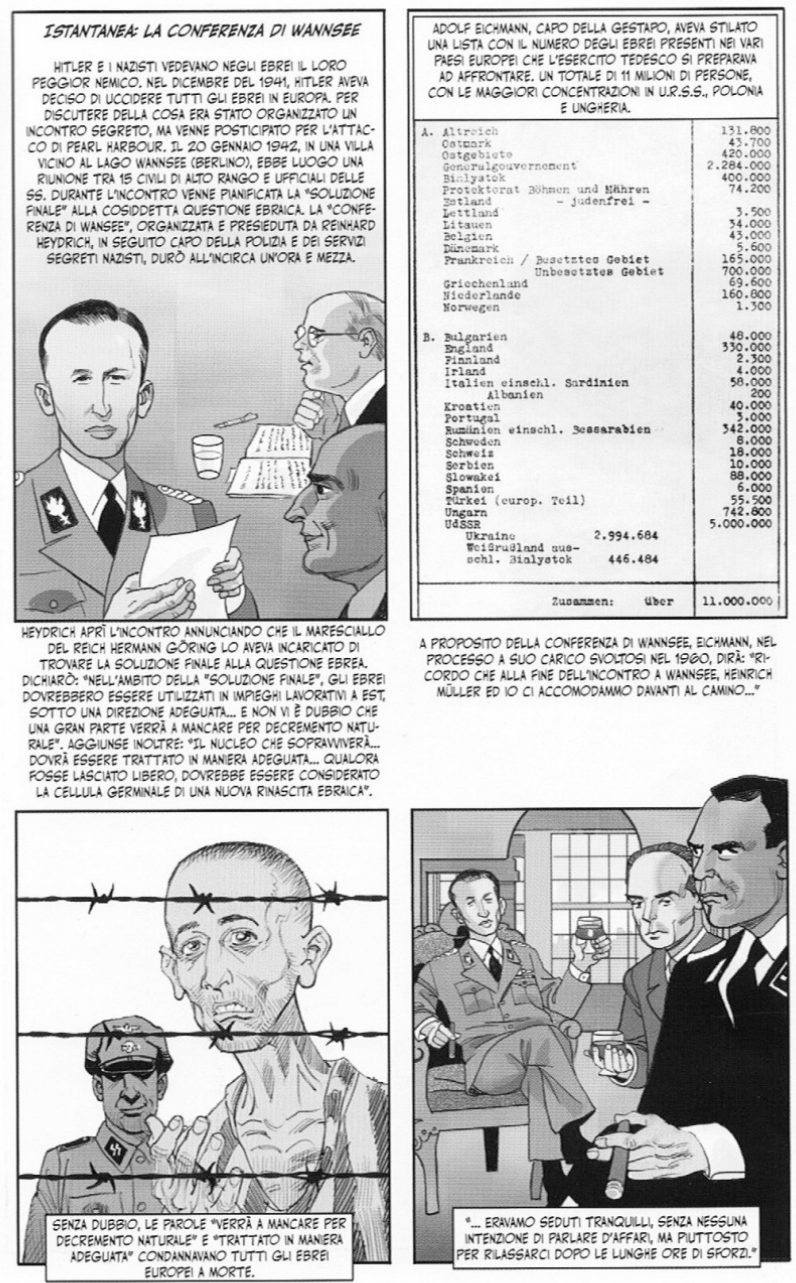

69

Fig. 5. Anne Frank. The Anne Frank House Authorized Graphic Biography. Tavola p. 69 (c) Rizzoli Lizard (2010). 
Un altro passaggio interessante è segnato dal Capitolo 6: Il diario. È qui che ha inizio il tempo del rifugio e, appunto, della scrittura del Diario, che Anne riceve in dono per il suo tredicesimo compleanno, come riporta il testo verbale: "Il 12 giugno 1942".

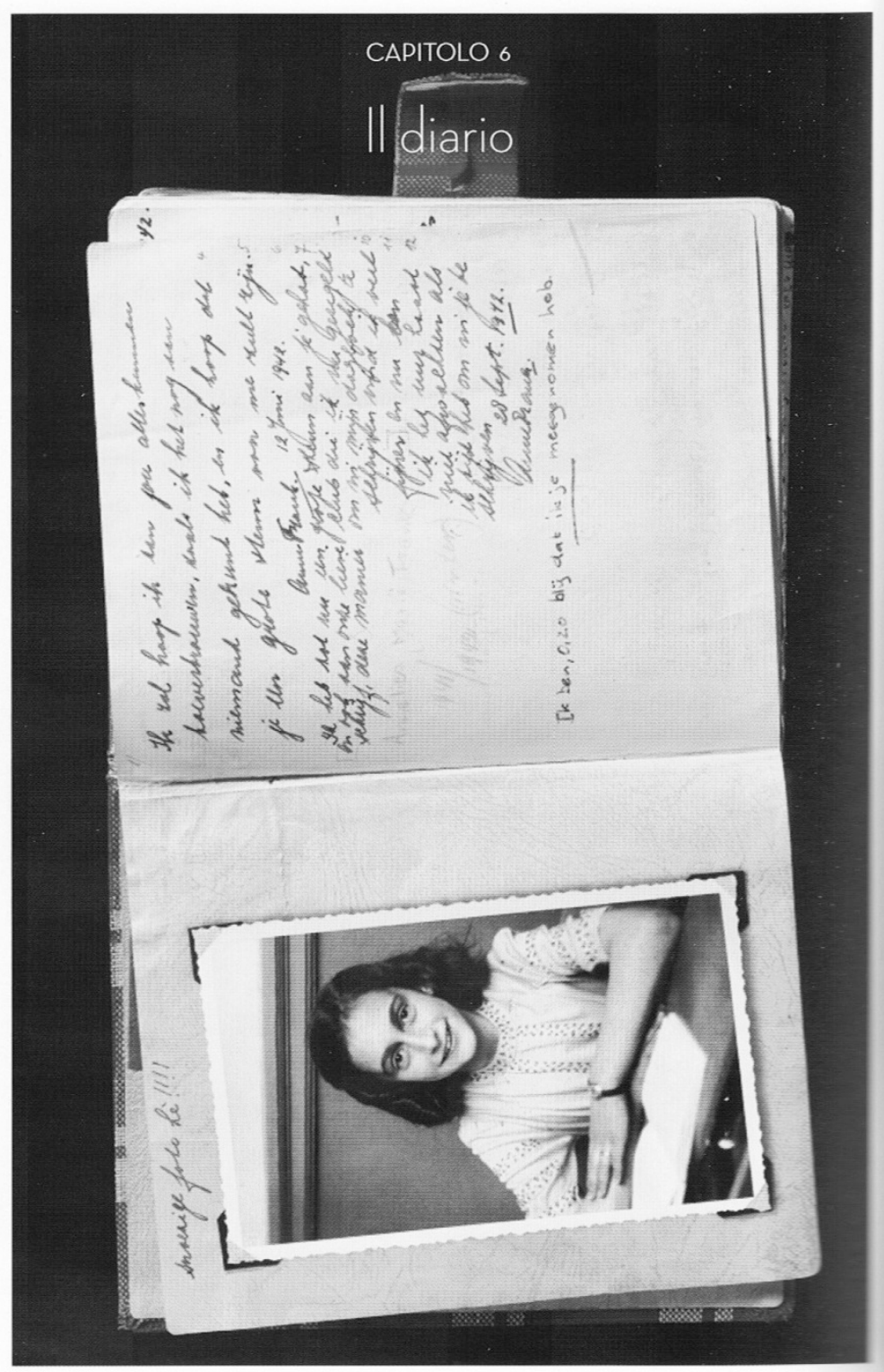

Fig 6. Anne Frank. The Anne Frank House Authorized Graphic Biography. Tavola p. 72 (c) Rizzoli Lizard (2010). 
Ciò che distingue questa apertura al capitolo dalle altre, è l'innesto dell'immagine fotografica. Si tratta della fotografia del diario di Anne, sulla cui pagina è incollata una sua foto (Fig. 6). Oltre a rappresentare un riferimento intertestuale, al Diario, agli archivi, alla Casa Museo, al Museo Virtuale, e dunque sul suo valore transmediale, questa tavola è una metanarrazione fotografica, che mette in relazione, inscritti all'interno dell'enunciato, il soggetto guardante e il soggetto guardato. Attraverso Barthes, possiamo rivedere il rapporto tra graphic novel e fotografia, nel quadro del discorso biografico:

La fotografia può dirmelo, molto meglio dei ritratti dipinti. Essa mi permette di accedere a un infra-sapere; mi fornisce una collezione di oggetti parziali e può solleticare in me un certo qual feticismo: infatti, vi è un «io» che ama il sapere, che prova nei suoi confronti come un gusto amoroso. Nello stesso modo, io amo certi aspetti biografici che, nella vita di uno scrittore, mi affascinano al pari di certe fotografie; ho chiamato questi aspetti "biografemi"; la Fotografia ha con la Storia lo stesso rapporto che il biografema ha con la biografia (1980, trad. it. 1980: 30).

Essa restituisce i particolari fondanti il sapere etnologico, ma quando affiancata al fumetto, ne sottolinea la principale differenza data da quellillusione di movimento, dalle forme ritmiche di percezione, di pensiero e di azione, che l'arte sequenziale costruisce abilmente.

II racconto della vita nel rifugio procede non attraverso le parole di Anne - come siamo abituati a leggere dal suo Diario -, ma con quelle del narratore extradiegetico che da onnisciente, con identità celata, diviene un soggetto riconoscibile in quanto a sua volta lettore e reporter dei contenuti del Diario di Anne. La descrizione verbo-visiva minuziosa dell'edificio di Prinsengracht $263^{8}$ ad Amsterdam, dei locali annessi sul retro, in cui la famiglia Frank trova rifugio insieme ai van Pels e, successivamente, a Fritz Pfeffer si affianca al racconto della discrezione che ne fece Anne. In una tavola in particolare, sul piano visivo,troviamo una successione di inquadraturein soggettiva, dove l'immagine sembra quasi il risultato di una rielaborazione grafica di una fotografia, mentre il narratore ricorda come Anne la descrisse nel suo diario.La ricerca di un effetto sfocato sembra coincidere con la messa in risalto di alcuni oggetti e, con essi, di alcuni dettagli, come la postazione di scrittura di Anne (trad. it. 2010: 82).

Il Capitolo 9, quello della cattura, racconta la deportazione, il campo di concentramento, e segna la fine del Diario e di Anne. Il Capitolo 10 ha inizio con la liberazione dei sopravvissuti ai campi nazisti e narra le vicende dell'unico superstite della famiglia Frank, Otto, che ritrovato il Diario di Anne decide dapprima di tradurlo e riscriverne alcuni passi per la sua famiglia in

8 L'edificio è stato aperto al pubblico nel 1960 è oggi è un museo. 
Svizzera, e successivamente di pubblicarlo. In questo capitolo, si assiste a uno spostamento dalla narrazione della storia collettiva del Conflitto e individualedella vita della famiglia Frank e di Anne, al progetto di costruzione di una memoria collettiva, che passa per la pubblicazione del Diario e per l'apertura del Museo. A conferma di ciò, anche qui ritroviamo l'innesto della fotografia come documento di ciò che fu il rifugio prima che diventasse l'Anne Frank Haus (Fig. 7). Infine la Cronologia, sviluppata su sei pagine, le cui intestazioni riportano frasi di Otto, va dal matrimonio tra Edith e Otto alla sua morte nel 1980; la Cronologia impiega la fotografia nella ricostruzione di una memoria individuale, fatta di immagini di famiglia, documenti di riconoscimento,e attestazioni pubbliche - come loscudo di David con la scritta Jood $^{9}$, che gli ebrei dai sei anni in su dovevano portare sui vestiti a partire dal 12 giugno 1942 -,affiancandola ai punti di una storia collettiva, resa invece a livello verbale, quella dell'ascesa del nazismo e della tragedia della Shoah, che integra e si alterna ai momenti significativi della famiglia Frank e degli altri clandestini del rifugio.

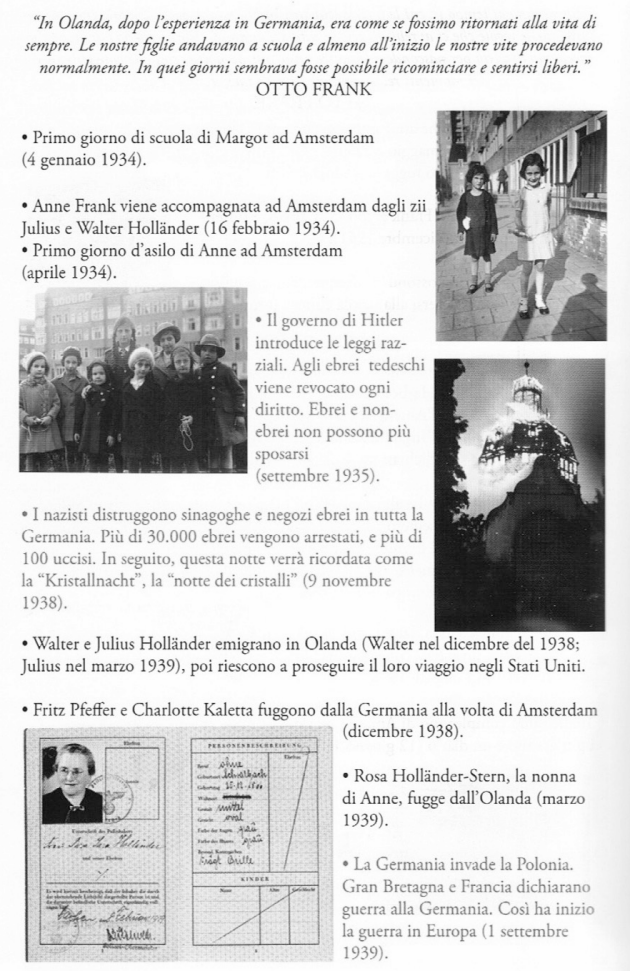

Fig. 7. Anne Frank. The Anne Frank House Authorized Graphic Biography. Tavola p. 152 ○) Rizzoli Lizard (2010).

9 Giudeo in olandese. 


\section{MEMORIA FRAMMENTATA E DOCUMENTI SUPPLENTI. IL SAPERE E IL CREDERE}

In Anne Frank, il documento ha connotazioni positive e assume un ruolo centrale nello svolgimento delle vicende narrate. Dinanzi all'abbondanza di documenti, si fa strada il bisogno di riassumere e di collegare i materiali in una sorta di enciclopedia globale e continua. II concetto di bricoleur ci viene in aiuto. Se pensiamo al significato che ne propone Lévi-Strauss, quando vi riconosce colui che parte da un segno esistente eraggruppa un numerodi "blocchi previncolati" di significazione, possiamo provare a riconoscere in esso il lavoro compiuto dal graphic novel, ancor più nel caso specifico di Anne Frank. Esso integra elementi preesistenti (biografie, diari, documenti d'archivio ecc.) in una rete di relazioni, e a partire da questa realizza una struttura significante. L'interrogativo cruciale ruota dunque intorno al creder-vero e alla relazione tra sapere $e$ credere. La distinzione tra racconto storico e racconto di finzione implica un differente modello di relazione tra il testo e il suo destinatario. Se nel primo caso, quest'ultimo assume un fare indagatore, nel secondo, dopo una prima esitazione, cede al creder-vero sollecitato dal racconto di finzione, abbandonando il sospetto, per mezzo del dispositivo dell'immaginazione. Nel graphic novel contemporaneo il confine tra i due si fa sempre più permeabile, raggiungendo un certo grado di versamento. Come ulteriore spunto, lo stesso Ricoeur inserisce in una nota il riferimento a Maus di Art Spiegelman, chiedendo e chiedendosi perché una modalità di racconto come quella di Maus non possa essere considerata appropriata (Ivi: 368 , nota 40).

\subsection{L'OPERA DI CULTO, L'ESPERIENZA IMMERSIVA E IL PARADOSSO DEL DOCUMENTO}

Il tema della Shoah è stato ampiamente sviluppato dal fumetto. Esemplare in tal senso è Maus di Art Spiegelman. Al tempo stesso, altre forme espressive hanno trattato questi temi, spesso a partire dalla costruzione di un punto vista individuale. Lo stesso Diario di Anne Frank, così come la produzione letteraria di Primo Levi. Ma cosa fa di un testo come Mause dello stesso Diario di Anne Frank un'opera di culto? Ciò che li accomuna è l'aderenza ai meccanismi di costruzione, organizzazione e conservazione della memoria collettiva; ciò che ne fa un'opera di culto è il fatto che tale funzionamento passi attraverso un'istanza individuale, quella dell'lo testimone, tanto da far nascere quasi un movimento artistico del graphic novel pseudo autobiografico, e richiamare diversi tipi di lettori. Inoltre la tensione generata tra l'evento traumatico e i modi del discorso ironico, assenti in Anne Frank. La biografia a fumetti, genera una tensione che consiste in un investimento di valori.

E allora un'opera come L'ombra delle torri di Art Spiegelman si fa documento attraverso un'istanza individuale in grado di rielaborare una memoria collettiva, alla luce di una conoscenza 
condivisa con il lettore. Essa passa sia attraverso la citazione al fumetto d'epoca sia attraverso la ricostruzione dell'immaginario mediale sugli eventi dell'11 settembre 2001 (Greco, 2015).

Potremmo parlare dell'attribuzione di una sorta di funzione mitica a questi testi, che proviene dall'impiego di un metodo di inserimento dell'irrazionalità della turbolenza affettiva nella razionalità del discorso giornalistico. Anne Frank si inserisce all'interno di un discorso più ampio, centrato sull'essenziale. Prendendo come suggerimento quanto dice Lévi-Strauss sull'importanza per il visitatore che gli venga mostrato il letto dove dormì Van Gogh, l'attenzione si sposta dal sapere al credere (1962, trad. it. 2010: 264). Nel caso in oggetto, ad esempio nella visita al rifugio di Anne Frank e all'esperienza immersiva veicolata dalla visita virtuale sul web, la sollecitazione della componente affettiva attiva un fare interpretativo che si sposta dal sapere per approdare al credere. Linterrogativo cruciale riguarda quindi l'affidabilità della testimonianza dell'lo e quella del documento, ovvero una bilancia tra fiducia e sospetto. Ed è proprio quest'ultimo, secondo Ricoeur, a percorrere tutto il ciclo della testimonianza, e dunque a diventare costitutivo dell'atto di testimoniare stesso. Ciò vuol dire che il sospetto caratterizza la testimonianza in tre fasi, nell'attimo in cui il testimone assiste ai fatti, nella reiterazione del ricordo di ciò che ha visto e ha vissuto e, infine, nella sua fase dichiarativa e narrativa, ovvero quando restituisce sottoforma di racconto un vissuto e un visto. Secondo questa considerazione, la certificazione della testimonianza si ottiene, dunque, solo quando il fare interpretativo dell'enunciatario sviluppa un credere. C'è un punto in particolare, di quelli individuati da Ricoeur, quando definisce le componenti della testimonianza, che sembra rispondere di questa condizione. Ed è l'autodesignazione del soggetto testimone, prerogativa della testimonianza, essa consiste l'attribuzione di ricordo a se stessi (lo c'ero). Dunque, fattore importante è il dichiararsi del testimone in quanto testimone. Un triplice deittico segna l'autodesignazione: la prima persona del singolare, il tempo passato del verbo e il là rispetto a qua. Questa struttura non è altro che il gioco dell'enunciazione enunciata e dei dispositivi del débrayage e dell'embrayage. In questo caso avremo un "io" che racconta di sé, ma in un altro tempo e in un altro luogo, dunque un non ora e non qui. L'autoreferenzialità a volte è marcata da enunciati introduttivi, come prefazioni. Tali asserzioni collegano, dice Ricoeur, la testimonianza puntuale alla storia di una vita, pensiamo alla prefazione ad Anne Frank. La biografia a fumetti, ed è per questo che a volte può capitare che ciò che riveste importanza per il testimone non abbia stesso valore per chi riceve la testimonianza.

\section{PER CONCLUDERE}

Se generi come l'autobiografia, le memorie, i reportage, i diari hanno un valore contrattuale più obbligante rispetto ad altri, poichél'"Io"si impegna in qualità di testimone di fronte al "tu", un testo come Anne Frank. La biografia a fumetti, pur nella presenza di 
documenti e fonti storiche decisive per la ricostruzione della Storia, rappresenta un caso interessante perché a causa del già visto troppe volte della storia di Anne, e del Diario, divenuto esso stesso documento, rischia di richiudersi nel guscio delle testimonianze al limite. Quella testimonianza straordinaria diventerebbe allora al limite non solo per l'impossibilità di condividere un'inumanità che non rientra nell'esperienza ordinaria di chi la riceve. La reiterazione, in questo caso, non ne rafforza la credibilità e l'affidabilità, ma l'adagia sul terreno di un passato elaborato, sino alla sua transizione a Documento/ Monumento, dove il primo, sospeso nel limbo delle commemorazioni collettive e rinviato, acquisisce la stessa tendenza a tergiversare del monumento. Tuttavia, I'analisi, che non si intendecompiuta, ma fa parte di una ricerca più ampia che intendo proseguire, ha fatto emergere quella capacità del graphic novel di risemantizzare il concetto stesso di documento, la cui efficacia simbolica, nel caso specifico in esame, rischia di dileguarsi e trasformare un esercizio di memoria in vezzo. Lo fa riempiendo i vuoti, per completare una storia attraverso il dispositivo dell'immaginazione, come in una metafiction (Waugh, 1984). Qui il testo devia rispetto alla storia fondata sul documento esistente, passando da un'illusione di realtà allo svelamento dell'illusione, portandoci a vedere in un tumulto affettivo ciò in cui finiamo per credere, il momento in cui Anne e la sorella sono prigioniere nei campi nazisti in cui perderanno la vita.

Anne Frank. La biografia a fumettiè sì una testimonianza, ed essa stessa si fa memoria storica, documento, nel suo bisogno di rimettere a posto i pezzi, di lavorare sulla relazione tra i frammenti, al fine di ri-generare il ricordo, trovare nuovi modi di autenticare il reale, ma al tempo stesso, per questa sua necessità, essa è espressione e provadi una trasformazione culturaledi ricerca di un continuo nella discontinuità.

\section{RIFERIMENTI BIBLIOGRAFICI}

BARTHES, R. (1968). "L'effet de réel". Communications 11. Trad. it. "L'effetto di reale". In II brusio della lingua, 151-159. Torino: Einaudi, 1988.

(1973).“Variations sur l'écriture”. In Oeuvres Complètes, t. II, Paris: Seuil, 1994. Trad. it.

"Variazioni sullascrittura", in Id., Variazioni sulla scrittura seguite da ll piacere del testo, 1999.

(1980). La chambre claire. Note sur la photographie. Paris: Gallimard-Seuil . Trad. it. La camera chiara. Nota sulla fotografia. Torino: Einaudi, 1980.

BARROUX, (2011). On les aura! Carnet de guerre d'un poilu (août, septembre1914), Paris: Seuil (Collection Album Jeunesse).

Cólon, E. \& Jacobson, S. (2010). Anne Frank: The Anne Frank House Authorized Graphic Biography. New York: Hill \& Wang Pub. Trad. it. Anne Frank. La biografia a fumetti. Milano: Rizzoli Lizard. FRANK, A. (1947). Het Achterhuis, Dagboekbrieven 14 juni 1942 - 1 augustus 1944. Amsterdam. GENETTE, G. (1987). Seuils. Paris: Seuil. Trad. it. Soglie. Torino: Einaudi, 1989. 


\section{CRISTINA GRECO}

GREIMAS, A. J. (1983c). Du sens II - Essais sémiotiques. Paris: Seuil. Trad. it. Del senso 2, P. Magli e M. P. Pozzato (a cura). Milano: Bompiani, 1985.

(1987). De l'imperfetion. Paris: Édition Pierre Fanlac. Trad. it. Dell'imperfezione. Palermo: Sellerio, 1988; ed. cons. Sellerio, 2004.

GRECO, C. (2009). Con la china in testa. Fumetto e memoria culturale. Per una lettura di Maus e

Palestina. Padova: Libreriauniversitaria.it.

(2014). Graphic novel. Confini e forme inedite nel sistema attuale dei generi. Roma: Nuova

Cultura.

(2015). “Finzione, storia e autenticazione del reale.Una lettura de L'ombra delle torri". E|C, 24 settembre.

GUIBERT, E.; LEFEVRE, D. \& LEMERCIER, F. (2008). Le Photographe. Paris: Dupuis. Trad. it. II Fotografo. Bologna: Coconino Press.

LE GOFF, J. (1978). “Documento/Monumento". In Enciclopedia, vol. V, 34-48. Torino: Einaudi.

LÉVI-STRAUSS, C. (1962). La pensée sauvage. Paris: Plon. Trad. it. Il pensiero selvaggio. Milano: II Saggiatore/Net, 2003, ed. cons. 2010.

LOTMAN, J. (1984). "O semiosfere". Töid märgisüsteemide alalt/Trudy po znakovym sistemam /

Sign Systems Studies 17, 5-23. Trad. it. La semiosfera. L'asimmetria e il dialogo nelle strutture pensanti. Venezia: Marsilio, 1985.

PEZZINI, I. (2007). II testo galeotto. La lettura come pratica efficace. Roma: Meltemi.

RICOEUR, P. (1984). Temps et récit II. La configuration dans le récit de fiction. Paris : Éditions du

Seuil, 1984. Trad. it. Tempo e Racconto. Volume 2. La configurazione nel racconto di finzione.

Milano: Jaca Book, 1999.

(2000). La mémoire, I'histoire, l'oubli. Paris: Seuil. Trad. it. La memoria, la storia, l'oblio.

Milano: Cortina, 2004.

SACCO, Joe (1993). Palestine, Vol. 1: A Nation Occupied. Fantagraphics Books.

SANSONE, C. \& TOTA, A. (2012). Palacinche. Storia di un'esule fiumana. Roma: Fandango.

SPIEGELMAN, A. (1986). Maus I: A Survivor's Tale: My Father Bleeds History. New York: Pantheon

Books.

(1992). Maus II: A Survivor's Tale: And Here My Troubles Began. New York: Pantheon Books. (2004). In The Shadow of No Tower. New York: Pantheon Books. Trad. it. L'ombra delle torri. Milano: Einaudi, 2014.

WAUGH, P. (1984). Metafiction: The Theory and Practice of Self-Conscious Fiction. London: Routledge. 